\title{
A PILOT STUDY ON THE PREVALENCE OF ARTIFICIAL INTELLIGENCE IN CANADIAN ENGINEERING DESIGN CURRICULA
}

\author{
Pranav Milind Khanolkar ${ }^{l}$, Mohammed Gad ${ }^{2}$, Jessica Liao ${ }^{2}$, Ada Hurst ${ }^{2}$, Alison Olechowski ${ }^{1}$ \\ ${ }^{l}$ Department of Mechanical and Industrial Engineering, University of Toronto \\ ${ }^{2}$ Department of Management Sciences, University of Waterloo \\ pranavm.khanolkar@mail.utoronto.ca, olechowski@mie.utoronto.ca
}

\begin{abstract}
Recent advances in artificial intelligence (AI) have shed light on the potential uses and applications of AI tools in engineering design. However, the aspiration of a fully automated engineering design process still seems out of reach of AI's current capabilities, and therefore, the need for human expertise and cognitive skills persists. Nonetheless, a collaborative design process that emphasizes and uses the strengths of both AI and human engineers is an appealing direction for AI in design. To uncover the current applications of AI, the authors review literature pertaining to AI applications in design research and engineering practice. This highlights the importance of integrating AI education into engineering design curricula in post-secondary institutions. Next, a pilot study assessment of undergraduate mechanical engineering course descriptions at the University of Waterloo and University of Toronto reveals that only one out of a total of 153 courses provides both AI and design-related knowledge together in a course. This result identifies possible gaps in Canadian engineering curricula and potential deficiencies in the skills of graduating Canadian engineers.
\end{abstract}

Keywords: artificial intelligence, engineering design, design education, design curricula

\section{INTRODUCTION}

Design is one of the fundamental practices of engineering that distinguish engineering from other fields [1]. Engineering design is the iterative process of identifying a need, and conceiving, building and testing a solution to meet that need. The components, systems, and processes designed by engineers in turn interact with and impact social, economic, and environmental systems. As such, engineering design stands as a primary focus of undergraduate engineering education in Canada and worldwide.

Despite its central role, there seem to be many challenges in articulating why design is hard to learn and harder to teach [1]. As Evans et al. [2] said, "[design] seems to occupy the top drawer of a Pandora's box of controversial curriculum matters," an indication of design education's ambiguous nature. It is crucial that design education is delivered relevant to current technologies, with the aim of equipping all students with the skills required to excel as designers. As a result, one of the strategies suggested by Butt et al. [3] to tackle this challenge is by adopting a transdisciplinary approach to engineering design education. This will not only expose students to multiple fields applicable to design but also facilitate effective communication between engineers through their familiarity with different industry standards and terminologies.

One of the most impactful recent developments in engineering design practice is the adaptation and application of AI-based tools. Due to the advent of digitalization in a majority of industries, the number of artificial intelligence (AI) applications has proliferated exponentially [4], [5]. This massive increase in demand for AI has subsequently led to the increase of accessible data repositories, which in turn encouraged the development, processing and analysis of novel AI-based applications [6]-[8]. The field of engineering design has proven to be a rich context for various applications of AI (highlighted in section 2) where its capabilities can be pushed to their limits, with a potential prospect of a balanced human-AI collaborative engineering design process, as depicted in Figure 1. Although computers have become highly effective at cognitive applications such as image processing, facial recognition and language processing, humans still excel in tasks requiring creative reasoning and interpersonal empathy compared to computers. A balanced human-AI collaborative engineering design process can leverage the computational power of AI along with the cognitive skills of humans to provide a rich and efficient design experience.

As AI becomes more integrated into our society, engineering students are also expected to become equipped with AI literacy, occupational-related skills to work with AI. The students are expected to have an awareness of not only the benefits but also the limitations and possible risks of improper applications that AI poses on stakeholders and society. A recent study found that only 15 out of 81 Canadian public universities offer moderate to broad AI education [9]; yet the growing role of AI-based tools and techniques in engineering design applications along with their limitations and risks, warrants a review of current 


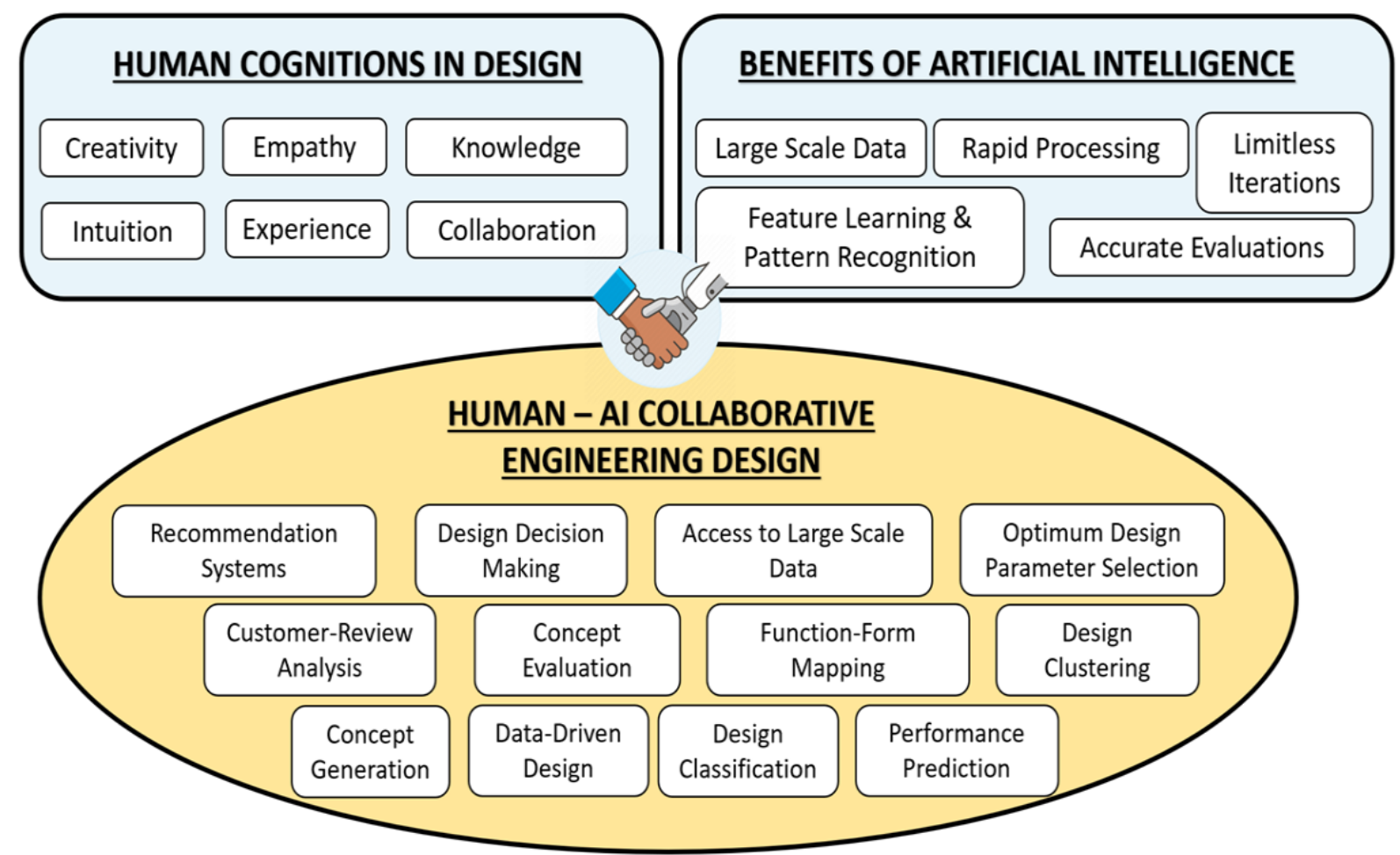

Figure 1: Some applications and benefits of human-Al collaboration with combined skills and benefits of humans and Al.

engineering design education. Are engineering students being exposed to AI-based tools in engineering design education? And if so, what is the extent and nature of this exposure?

While researchers have highlighted the necessity to incorporate $\mathrm{AI}$ in engineering education curriculum based on the emergence of the Industry 4.0 phenomenon [10][12], a gap exists specifically to investigate the current prevalence of $\mathrm{AI}$ in engineering design education in the curricula of Canadian universities. In this exploratory paper, we review current applications of $\mathrm{AI}$ in the field of engineering design. Further, in a case study of mechanical engineering curricula at the authors' affiliated universities, we review engineering design-based coursework with the purpose of benchmarking the current level of penetration of AI topics in engineering design education.

\section{ARTIFICIAL INTELLIGENCE IN ENGINEERING DESIGN}

\subsection{What is Artificial Intelligence?}

Due to its rapid progress and diverse nature, the precise definition for AI has been up for debate. Most definitions of AI can be classified under one of four categories: systems that think and reason like humans, systems that think and reason rationally, systems that behave like humans, and systems that behave rationally [13]. A humancentered view of $\mathrm{AI}$ is more common in empirical science fields, whereas a rationalist view is more common in mathematics and engineering fields [13].

AI is a diverse and versatile technology with applications in many fields, from medicine to art to security and much more. Subsets of AI include Natural Language Processing (NLP) which bridges the gap between 'computer' language and 'human' language, via knowledge representation which stores information for use in automated reasoning to answer questions and draw conclusions. Another subset of AI is machine learning, which comprises adaptive algorithms that detect and extrapolate patterns [13]. Machine learning, one of the most widely used AI tools, has the ability to learn features and patterns in data through statistical analysis and perform predictions, classifications, or clustering of data similar to which it has been trained [14]. Moreover, deep learning, a subset of machine learning, takes machine learning a step further with artificial neural networks, which enable computers to perform much more complex tasks such as facial and object detection, handwriting recognition, image and video analysis, and others [15], [16].

AI's great potential and versatility have led to its use in many other fields and industries such as robotics, recommendation systems, computer vision, etc., which expand the horizons of AI applications [13]. 


\subsection{AI in Engineering Design: Literature Review}

Engineering design researchers utilize AI-based algorithms methods, especially machine learning, for rapid design data learning and processing [17]-[19] and have achieved successful results in their research contributions. Such contributions include evaluating design concepts [20], decision making for design support systems [21], design for additive manufacturing [22], predicting strain fields in microstructure designs [23], predicting performance of design based on its shape and vice-versa [24], material selection for sustainable product design [25] etc. Certain applications of AI that have proven efficient in analyzing computer-aided design (CAD) data include predicting the function of CAD model from its form [26], suitable feature-removal in CAD models for simulations [27], and CAD design shape matching [28]. Certain studies [29]-[31] potentially offer common ground between human designers and AI to provide opportunities for hybrid human-agent design. For example, Raina et al. [32] proposed a two-step framework using deep learning constructs and visual interface interactions for AI agents to imitate human design strategies from observation and effectively create feasible and efficient designs. Machine learning classification algorithms have been proven useful in categorizing customers' product reviews based on the product's function, form, service or behaviour [33]. Wisthoff et al. [34] have utilized artificial neural networks to predict the life cycle assessment of a product design from its attributes.

Such remarkable contributions, along with a basic understanding of AI and machine learning, provide inspiration for aspirant designers to harness such skills for design and innovation. Researchers predict that AI will change how engineers work rather than replace engineers [35], [36]. By allowing the tedious and often timeconsuming tasks involved in engineering design to be performed by AI tools such as neural networks, machine learning, computational agents and others, design engineers will have more opportunities to solve problems creatively.

\subsection{Limitations of AI in Engineering Design}

Having reviewed the positive potential for AI's integration with engineering design, it is important to highlight the limitations of $\mathrm{AI}$ and how they relate to engineering design.

Broadly speaking, there are still many skills that computational systems have yet to master and which are recognized as essential to engineering design. AI cannot effectively learn and replicate creativity [37], interpersonal empathy [38], [39], intuition [40], knowledge consolidation or persuasion for problem-solving, or to coordinate and lead teams [41], [42]. AI cannot fully replace human designers because of their lack of creativity and agility, which is needed to solve complex engineering design problems [43]. Computerizing creativity is limited by the programmer's ability to define the creative values clearly to be encoded into an algorithm [44]. Unfortunately, creativity is a skill that student designers are lacking [45]. This means that AI is likely unable to aid novices to be more creative in their design.

A study by Zhang et al. [46] reveals that AI negatively impacts the performance of high-performing design teams by lowering the mental demand of the human designers since they tend to overestimate their control over uncontrollable events.

The use of AI is also associated with risks and challenges such as bias, deception, data control and privacy, displacement of jobs and skills, military and social control, and inequality. In fact, Rich \& Gureckis [47] make the argument that humans and AI exhibit many of the same limitations that impede unbiased human judgment. These biases in AI come about from different sources, such as small and incomplete datasets, biased inference processes, and many more [47].

\section{CASE STUDY}

The engineering design process necessitates a rich amalgamation of fundamental subject matter expertise as well as cognitive skills such as problem solving and creativity. A well-balanced engineering design curriculum should impart knowledge of traditional design methods and digitalization skills to best equip upcoming engineers and designers for impact in the world.

To understand the current prominence of AI integrated with engineering design, the authors reviewed the fouryear undergraduate mechanical engineering course curricula at the University of Waterloo and the University of Toronto. These universities were selected based on the authors' familiarity with the course curricula and affiliation. The field of mechanical engineering is considered to incorporate the engineering design domain and its related courses. Therefore, the mechanical engineering course curricula of the aforementioned universities were selected as case studies for this work. All core courses and technical electives that were stated on the program's webpages were considered for the study. It should be noted that non-mechanical engineering technical electives of the University of Waterloo programs were not considered since the options were not explicitly present on the curriculum webpage.

Web-scraping is an innovative tool used for extracting large amounts of data from webpages through automation [48]. Previous research works have successfully utilized web-scraping to extract information pertaining to online courses [49], [50]. As such, the names and descriptions of 73 courses from the University of Waterloo and 80 courses from the University of Toronto's undergraduate 
mechanical engineering programs were extracted from their respective webpage through the web-scraping process using BeautifulSoup4 Python library [51].

The authors curated a list of representative keywords obtained from the cited literature to filter the undergraduate courses that combined AI and design education. The extracted keywords included 31 unique words and phrases that represented AI, 43 unique words and phrases that represented engineering design, and 9 unique words and phrases representing both AI and design simultaneously. The keywords extracted from the literature are depicted in Figure 2. Subsequent to that, variations of these unique words were developed to account for the different word spelling and phrase structures such as the absence of a hyphen, abbreviations, capitalization, etc. A Python script was then written and executed on the web-scraped course information to filter out the courses that contained the keywords and phrases within their description.

Table 1. Filtered results of University of Toronto courses

\begin{tabular}{|c|c|c|c|c|}
\hline \multirow[b]{2}{*}{ Course Code } & \multirow[b]{2}{*}{ Course Name } & \multicolumn{3}{|c|}{ Number of keywords } \\
\hline & & AI & ED & $\begin{array}{c}\mathrm{AI} \\
+ \\
\mathrm{ED}\end{array}$ \\
\hline MSE401H1 & $\begin{array}{l}\text { Materials } \\
\text { Information in } \\
\text { Design }\end{array}$ & 2 & 1 & 0 \\
\hline \multicolumn{5}{|c|}{$\begin{array}{l}\text { AI: Keywords that represent Artificial Intelligence } \\
\text { ED: Keywords that represent Engineering Design } \\
\text { AI + ED: Keywords that represent Artificial Intelligence and } \\
\text { Engineering Design simultaneously }\end{array}$} \\
\hline \multicolumn{5}{|c|}{$\begin{array}{l}\text { Course Description: } \\
\text { This course presents approaches to composite and structural } \\
\text { design, and optimization, for components and products. Tools } \\
\text { for optimization, material property data analytics, and } \\
\text { structural simulation will be used. We will apply advanced } \\
\text { materials selection (and the CES materials database) to } \\
\text { product and component design, and hybrid (composite) } \\
\text { materials design. Composite mechanics theory and topology } \\
\text { optimization will be developed for structural optimization. } \\
\text { Finally, modern techniques including AI and machine } \\
\text { learning will be presented for aspects of materials selection, } \\
\text { composite design and structural optimization. Component } \\
\text { design decisions will include both material properties and the } \\
\text { capabilities of applicable fabrication processes, to identify the } \\
\text { material and process which best satisfy the design } \\
\text { requirements. }\end{array}$} \\
\hline
\end{tabular}

The results revealed that only one out of 80 undergraduate mechanical engineering course descriptions at the University of Toronto contained keywords that represented both AI and design separately (see Table 1). In comparison, none of the University of Waterloo's courses had any of the extracted keywords. The course offered by the University of Toronto was an elective course during the fourth year of the program: Materials Information in Design. The University of Toronto provides an introductory course on AI as an elective in the fourth year of the program, although it does not cover the AI applications in engineering design. These low numbers indicate the limited extent to which the students are exposed to the idea of AI and design simultaneously, through academic courses, and how many courses are available to them if they decide to pursue this field further.

Regardless of the lack of AI in engineering design courses offered at both programs, it was observed that both universities provide decent exposure to engineering design. The University of Toronto offers 21 courses with design content in their mechanical engineering undergraduate program indicated by the keywords such as CAD, prototyping, conceptual design, sketching, design process etc., whereas the University of Waterloo provides 9 courses indicated by the keywords such as creativity, detailed design, simulation, etc.

Furthermore, it is crucial to note that one of the factors that may affect the variation in course offering between the University of Toronto's and the University of Waterloo's mechanical engineering programs is the program structure. Mechatronics engineering, which is a design-focused engineering program, is offered as an option within the mechanical engineering program at the University of Toronto, whereas the same program is offered separately at the University of Waterloo. This difference in program structure might explain why the University of Waterloo's program course is small and less diverse than that of the University of Toronto.

\section{CONCLUSION}

In the age of digitalization, $\mathrm{AI}$ is increasingly integrated in the field of engineering design. A review of literature as stated in section 2 reveals the profound opportunities offered by AI to engineering designers. As highlighted in section 2, the advancements in AI necessitate that today's engineering graduates possess basic technical knowledge of AI and its current applications and potential uses. Although AI can out-perform human designers in dataintensive problem-solving applications, AI is still limited in tasks that require creative reasoning and interpersonal empathy. Therefore, it is vital for aspiring design engineers to accumulate various concepts pertaining to engineering design with AI and its current limitations through engineering education. Such knowledge obtained through education shall aid engineers to comprehend the relation between human design traits such as creativity, intuition, empathy, communication, and utilizing appropriate AI techniques. As AI becomes more prominent in the field of engineering design, it is integral that design education properly prepares students with the knowledge and skills to work with the opportunities and implications associated 


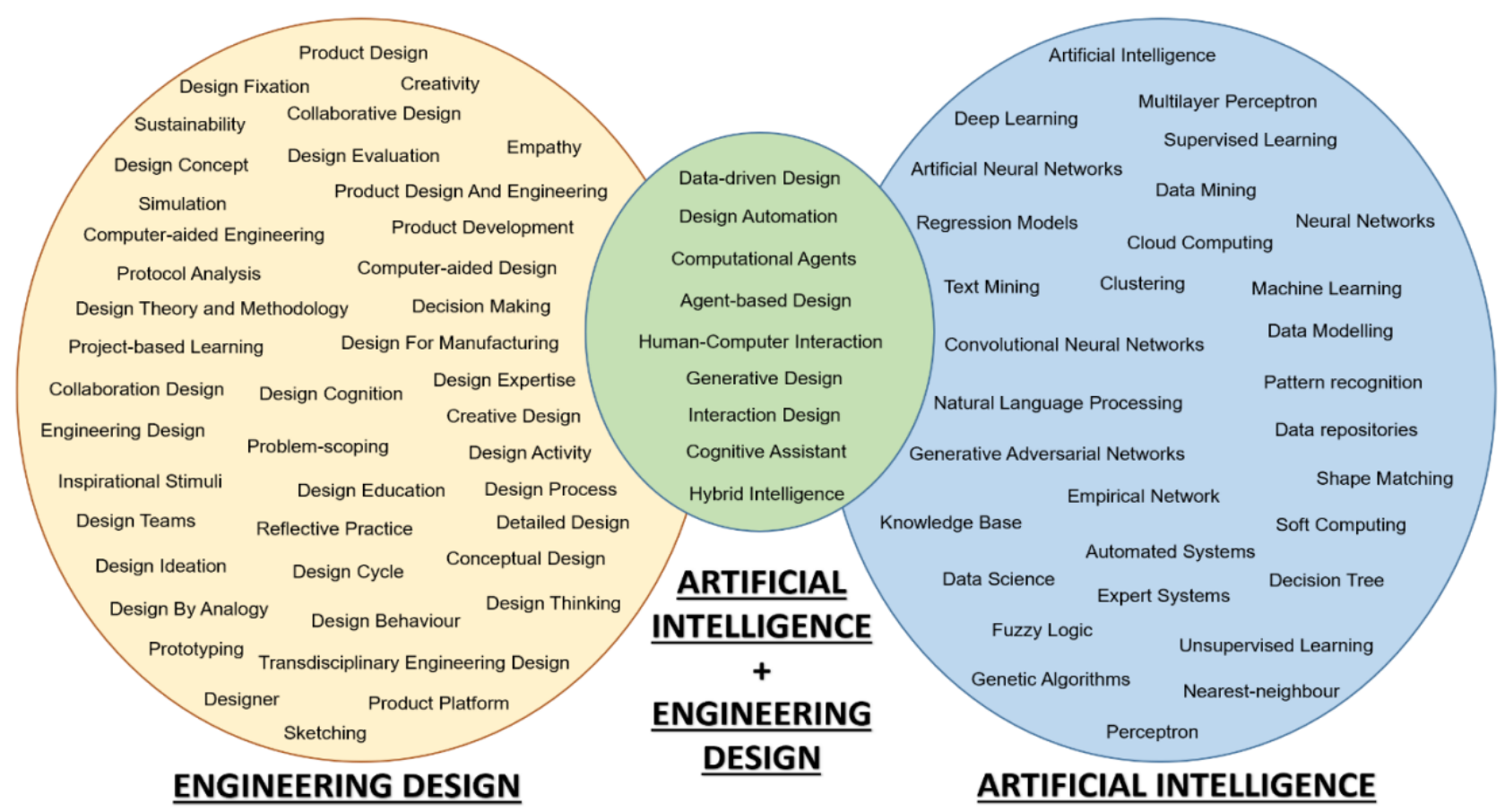

Figure 2: List of keywords extracted from literature that describe artificial intelligence, engineering design and both.

with AI. Providing a strong theoretical background and a practical understanding of AI topics such as machine learning, NLP, deep learning, etc., can enable students to utilize their creativity and intuition to develop novel AIbased approaches towards engineering design problems. Thus, besides updating themselves with recent AI applications in the industry, students can familiarize themselves with learning and applying $\mathrm{AI}$ and traditional design concepts together to solve existing and new design problems in an innovative fashion, leading them to become better designers.

A pilot study of the engineering course curricula at the University of Waterloo and University of Toronto was conducted to understand the current engineering design education. Results from the study indicate that the current engineering education does not offer adequate courses that teach both AI and engineering design. The lack of AI courses in engineering design curricula, along with the increase in AI applications in design research and industry, can be viewed as an opportunity for promoting courses that provide $\mathrm{AI}$ exposure to graduating engineers in the field of engineering design.

Engineering design education needs to teach AI skills, knowledge, societal and personal implications in all undergraduate and graduate programs to ensure that graduating engineering students have an adequate understanding of AI. Educators and education authorities should create implementation plans to incorporate $\mathrm{AI}$ in the engineering design curricula. As AI continues to change, it is important that educators are familiarized with AI. It is also recommended that the Canadian government work with educators and employers to define the competencies and learning outcomes necessary to work with AI.

\section{LIMITATIONS \& FUTURE WORK}

A limitation of the case study is the use of course descriptions to understand the current engineering design education. Course descriptions may not provide the most accurate portrayal of a course's learning outcomes and what the students actually learn. Course outlines may provide a more accurate representation of the courses.

The sample size also limits the case study since the courses present on the mechanical engineering curriculum webpages of both, University of Waterloo and the University of Toronto were only considered. As such, course descriptions were only extracted from these engineering undergraduate courses of these universities. Therefore, the results may not reflect the engineering design curricula provided by the engineering disciplines and the Canadian universities outside the scope of this study. A future study will be conducted to vastly expand the scope of analysis to university programs in Canada and internationally, to evaluate the actual prevalence and exposure of AI-related coursework in such post-secondary institutions.

Furthermore, the results of this study are limited by the capabilities of the Python script written to filter courses with the keywords present in their description. Synonyms of keywords were not considered in this study. Therefore, 
the method used to filter courses will be changed in future iterations.

So far, the work done in this paper paves the way for further research into assessing the success and efficacy of engineering undergraduate programs in Canada in equipping future engineers with the tools and skills required to keep up with and excel in uprising fields in design. The next step would be to widen the scope of the engineering programs assessed in research. A more accurate and representative conclusion can be obtained by including a more diverse range of engineering undergraduate and graduate programs from a wider range of Canadian universities. Furthermore, the same process can be applied to universities outside of Canada to be used as a control to assess the success of Canadian universities. Lastly, to overcome the limitations imposed by the use of keywords and the Python script, NLP can gather information and context from course descriptions, outlines, and available course material.

\section{Acknowledgements}

This work is co-funded by the Social Sciences and Humanities Research Council and the Government of Canada's Future Skills program. The work is also supported through funding by the Government of Canada's Student Work Placement Program.

\section{References}

[1] C. L. Dym, A. M. Agogino, O. Eris, D. D. Frey, and L. J. Leifer, "Engineering design thinking, teaching, and learning," 2005, doi: 10.1002/j.21689830.2005.tb00832.x.

[2] D. Evans, "Design in Engineering Education: Past Views of Future Directions.," Eng. Educ., 1990.

[3] M. Butt, A. Sharunova, M. Storga, Y. I. Khan, and A. J. Qureshi, "Transdisciplinary Engineering Design Education: Ontology for a Generic Product Design Process," 2018, doi: 10.1016/j.procir.2018.02.019.

[4] J. Lee, H. Davari, J. Singh, and V. Pandhare, "Industrial Artificial Intelligence for industry 4.0-based manufacturing systems," Manuf. Lett., 2018, doi: 10.1016/j.mfglet.2018.09.002.

[5] B. hu Li, B. cun Hou, W. tao Yu, X. bing Lu, and C. wei Yang, "Applications of artificial intelligence in intelligent manufacturing: a review," Frontiers of Information Technology and Electronic Engineering. 2017, doi: 10.1631/FITEE.1601885.

[6] Y. Roh, G. Heo, and S. E. Whang, "A survey on data collection for machine learning: A big data - AI integration perspective," arXiv. 2018, doi: 10.1109/tkde.2019.2946162.

[7] T. J. Marion and S. K. Fixson, "The Transformation of the Innovation Process: How Digital Tools are Changing Work, Collaboration, and Organizations in New Product Development," J. Prod. Innov. Manag., vol. 0, no. 0, pp. 1-24, 2020, doi: 10.1111/jpim. 12547.

[8] Y. Feng, Y. Zhao, H. Zheng, Z. Li, and J. Tan, "Data- driven product design toward intelligent manufacturing: A review," International Journal of Advanced Robotic Systems. 2020, doi: 10.1177/1729881420911257.

[9] D. Ticoll, "SKILLING CANADIANS FOR LEADERSHIP IN THE AI ECONOMY," 2020.

[10] R. Sakhapov and S. Absalyamova, "Fourth industrial revolution and the paradigm change in engineering education," 2018,

10.1051/matecconf/201824512003.

[11] L. Jeganathan, A. N. Khan, J. Kannan Raju, and S. Narayanasamy, "On a frame work of curriculum for engineering education 4.0," 2019, doi: 10.1109/WEEFGEDC.2018.8629629.

[12] S. Das, D. K. Kleinke, and D. Pistrui, "Reimagining engineering education: Does industry 4.0 need education 4.0?," 2020, doi: 10.18260/1-2--35136.

[13] S. Russell and P. Norvig, Artificial Intelligence A Modern Approach Third Edition. 2010.

[14] M. I. Jordan and T. M. Mitchell, "Machine learning: Trends, perspectives, and prospects," Science. 2015, doi: $10.1126 /$ science.aaa8415.

[15] Y. Lecun, Y. Bengio, and G. Hinton, "Deep learning," Nature. 2015, doi: 10.1038/nature14539.

[16] L. Deng and D. Yu, "Deep learning: Methods and applications," Foundations and Trends in Signal Processing. 2013, doi: 10.1561/2000000039.

[17] D. L. Grecu and D. C. Brown, "Dimensions of machine learning in design," Artif. Intell. Eng. Des. Anal. Manuf. AIEDAM, vol. 12, no. 2, pp. 117-121, 1998, doi: 10.1017/s0890060498122047.

[18] M. Lou Maher and H. li, "Learning design concepts using machine learning techniques," Artif. Intell. Eng. Des. Anal. Manuf., vol. 8, no. 2, pp. 95-111, 1994, doi: 10.1017/S0890060400000706.

[19] S. K. Sim and A. H. B. Duffy, "A foundation for machine learning in design," Artif. Intell. Eng. Des. Anal. Manuf. AIEDAM, vol. 12, no. 2, pp. 193-209, 1998, doi: 10.1017/s0890060498122096.

[20] B. Camburn, Y. He, S. Raviselvam, J. Luo, and K. Wood, "Machine Learning-Based Design Concept Evaluation," J. Mech. Des., 2020, doi: 10.1115/1.4045126.

[21] L. Romeo, J. Loncarski, M. Paolanti, G. Bocchini, A. Mancini, and E. Frontoni, "Machine learning-based design support system for the prediction of heterogeneous machine parameters in industry 4.0," Expert Syst. Appl., 2020, doi: 10.1016/j.eswa.2019.112869.

[22] G. Williams, N. A. Meisel, T. W. Simpson, and C. McComb, "Design repository effectiveness for 3D convolutional neural networks: Application to additive manufacturing," J. Mech. Des. Trans. ASME, 2019, doi: 10.1115/1.4044199.

[23] P. M. Khanolkar, C. C. McComb, and S. Basu, "Predicting elastic strain fields in defective microstructures using image colorization algorithms," Comput. Mater. Sci., 2021, doi: 10.1016/j.commatsci.2020.110068.

[24] C. McComb, "Toward the Rapid Design of Engineered Systems Through Deep Neural Networks," in Design Computing and Cognition '18, 2019.

[25] C. C. Zhou, G. F. Yin, and X. B. Hu, "Multi-objective 
optimization of material selection for sustainable products: Artificial neural networks and genetic algorithm approach," Mater. Des., 2009, doi: 10.1016/j.matdes.2008.06.006.

[26] M. L. Dering and C. S. Tucker, "A Convolutional Neural Network Model for Predicting a Product's Function, Given Its Form," J. Mech. Des. Trans. ASME, 2017, doi: 10.1115/1.4037309.

[27] F. Danglade, J. P. Pernot, and P. Véron, "On the use of Machine Learning to Defeature CAD Models for Simulation," Comput. Aided. Des. Appl., 2014, doi: 10.1080/16864360.2013.863510.

[28] C. Yiu Ip and W. C. Regli, "Content-Based Classification of CAD Models with Supervised Learning," Comput. Aided. Des. Appl., 2005, doi: 10.1080/16864360.2005.10738325.

[29] S. Lapp, K. Jablokow, and C. McComb, “KABOOM: an agent-based model for simulating cognitive style in team problem solving," Des. Sci., 2019, doi: 10.1017/dsj.2019.12.

[30] D. L. Poole and A. K. Mackworth, Artificial intelligence: Foundations of computational agents. 2010.

[31] A. Raina, C. McComb, and J. Cagan, "Design strategy transfer in cognitively-inspired agents," 2018, doi: 10.1115/DETC2018-85599.

[32] A. Raina, C. McComb, and J. Cagan, "Learning to Design From Humans: Imitating Human Designers Through Deep Learning," J. Mech. Des., vol. 141, no. 11, pp. 1-11, 2019, doi: 10.1115/1.4044256.

[33] A. Singh and C. S. Tucker, "A machine learning approach to product review disambiguation based on function, form and behavior classification," Decis. Support Syst., 2017, doi: 10.1016/j.dss.2017.03.007.

[34] A. Wisthoff, T. Huynh, V. Ferrero, and B. Dupont, "Quantifying the impact of sustainable product design decisions in the early design phase through machine learning," 2016, doi: 10.1115/DETC2016-59586.pdf.

[35] A. K. Noor, "Ai and the future of machine design," Mech. Eng., 2017, doi: 10.1115/1.2017-oct-2.

[36] A. Raina, J. Cagan, and C. McComb, "Transferring design strategies from human to computer and across design problems," J. Mech. Des. Trans. ASME, 2019, doi: $10.1115 / 1.4044258$.

[37] S. W. Hsiao and J. R. Chou, "A creativity-based design process for innovative product design," Int. J. Ind. Ergon., 2004, doi: 10.1016/j.ergon.2004.05.005.

[38] M. Kouprie and F. S. Visser, "A framework for empathy in design: Stepping into and out of the user's life," $J$. Eng. Des., 2009, doi: 10.1080/09544820902875033.

[39] J. Zhou, V. Phadnis, and A. Olechowski, "Analysis of designer emotions in collaborative and traditional computer-aided design," 2019, doi: 10.1115/DETC2019-98516.

[40] E. Salas, M. A. Rosen, and D. DiazGranados, "Expertise-based intuition and decision making in organizations," J. Manage., 2010, doi: 10.1177/0149206309350084.

[41] E. Brynjolfsson, T. Mitchell, and D. Rock, "What Can Machines Learn and What Does It Mean for Occupations and the Economy?," AEA Pap. Proc., 2018, doi: $10.1257 /$ pandp. 20181019.
[42] J. Liao, P. Hansen, and C. Chai, "A framework of artificial intelligence augmented design support," Human-Computer Interact., vol. 00, no. 00, pp. 1-34, 2020, doi: 10.1080/07370024.2020.1733576.

[43] B. Song et al., "TOWARD HYBRID TEAMS: A PLATFORM TO UNDERSTAND HUMANCOMPUTER COLLABORATION DURING THE DESIGN OF COMPLEX ENGINEERED SYSTEMS," Proc. Des. Soc. Des. Conf., 2020, doi: 10.1017/dsd.2020.68.

[44] M. Boden, The creative mind: Myths and mechanisms. Psychology Press, 2004.

[45] J. P. Goff, R. M., \& Terpenny, "Engineering design education - Core competencies," 2012.

[46] G. Zhang, A. Raina, J. Cagan, and C. McComb, "A cautionary tale about the impact of AI on human design teams," Des. Stud., 2021, doi: 10.1016/j.destud.2021.100990.

[47] A. S. Rich and T. M. Gureckis, "Lessons for artificial intelligence from the study of natural stupidity," Nature Machine Intelligence. 2019, doi: 10.1038/s42256-0190038-z.

[48] P. Matta, N. Sharma, D. Sharma, B. Pant, and S. Sharma, "Web scraping: Applications and scraping tools," Int. J. Adv. Trends Comput. Sci. Eng., 2020, doi: 10.30534/ijatcse/2020/185952020.

[49] S. Kagemann and S. Bansal, "MOOCLink: Building and utilizing linked data from Massive Open Online Courses," 2015, doi: 10.1109/ICOSC.2015.7050836.

[50] N. Piedra, J. Chicaiza, J. López, and E. Tovar, "Seeking open educational resources to compose massive open online courses in engineering education an approach based on linked open data," J. Univers. Comput. Sci., 2015.

[51] "Beautiful Soup Documentation - Beautiful Soup 4.9.0 documentation."

https://www.crummy.com/software/BeautifulSoup/bs4/ doc/ (accessed Mar. 07, 2021). 